\title{
The Application of Nuclear Medicine
}

\author{
Susatyo Pranoto, Giner Maslebu, Suryasatriya Trihandaru \\ Department of Physics, Faculty of Science and Mathematics, \\ Satya Wacana Christian University \\ Jl. Diponegoro 47, Salatiga \\ E-mail : supranumed@gmail.com
}

Received: 15 June 2015, Revised: 30 September 2016, Accepted: 10 April 2016

\begin{abstract}
Currently, the practice of nuclear medicine in modern countries comprises a large number of procedures. It is applied to study function of organs/body systems, to visualize, to characterize, and to quantify the functional state of lesions and for targeted radionuclide therapy. This overview presents all kinds of application in nuclear medicine services. Instrumentation and radioactive/radiolabeled substances are the basic components for application. Biotechnology contributes to the development and production of biomolecules used in radiopharmaceuticals. As a diagnostic modality, imaging depicts radioactivity distribution as a function of time. Hybrid imaging provides more precise localization and definition of le-sions as well as molecular imaging cross validation. Counting tests study invivo organ functions externally or assess analytes in the biologic samples. Radiopharmaceutical therapy can be applied directly into the lesion or targeted systemically. Nanotechnology facilitates targeting and opens the development of bimodal techniques. In addition, neutron application contributes to the advancement of nuclear medicine services, such as neutron activation analysis, neutron teletherapy and neutron capture therapy.
\end{abstract}

Keywords: nuclear medicine, biomolecules, analytes, nanotechnology, neutron application.

\section{INTRODUCTION}

Nuclear medicine (NM) is defined as a medical specialty which involves unsealed sources of ionizing radiation for diagnosis, therapy and study of diseases. Most procedures use radiopharmaceuticals, predominately for diagnostic activities[1]. Radiopharmaceuticals are radiolabeled targeting agents administered to the patients. They can be in the form of particles, chemicals or biologicals [2].

As a diagnostic modality, NM can detect lesions very early, because functional (phsiological/biochemical) changes precede structure/morphological abnormalities In diagnostic workup, it visualizes and characterizes lesions, whether they contain viable cells or not, as well as what, where and how fast specific biological processes are going on. NM is also utilized to study organ/body system functions, such as performance, perfusion, tube/ tract patency, gene expression, etc [3].
The existence of therapeutic modality improves and advances the quality of patient treatments, particularly, in cancer management. NM shares roles with other medical modalities [4]. Each modality is not competitive, but complementary to the others.

The roles of NM include : identification of diseases; prognostication and risk stratification; monitoring the course of diseases; adjunctive, optional and choice of therapy; follow up therapeutic outcomes. NM can be used to decide whether the treatment must go on or stop, just because, unlike radiologic imaging, it can visually differentiate viable lesions from necrotic/scar tissues and granulation [5].

\section{PATHOBIOLOGY}

A lesion is a site containing functionally/ structurally abnormal cells. Various processes may take place such as: neoplasia, inflammation, infection, trauma, degeneration, cell dying, 
etc. Each process can be identified by certain radiopharmaceuticals, such as: neoplastic proliferation by F18-FLT, glycolysis by F18FDG, inflammation by Tc99m-fanole-somab, brain degeneration by C11-PK11195, apoptosis by Tc99m-annexin, infection by Tc99mciprofloxacin and so on [4,2].

\section{RADIOPHARMACEUTICALS}

A radiopharmaceutical usually contains a radionuclide (label) that is attached to its targeting molecule (vector) [4]. Few radionuclides do not require vectors, such as: I-131, Tc-99m, P-32, Sr-89, Ga-67. I-131, Tc-99m go to thyroid; P-32, Sr-89 respectively to prolifera-ting cells and bones; Ga-67 to neoplasma or inflammation. Radiopharmaceuticals interact with their respective targets at molecular level. Targets may be molecules or biochemical pathways. Non-biomolecular radiopharmaceuticals can be divided in 2 types, namely:

- Inorganic radiopharmaceuticals : P-32, Tc99m-MAA, Tc99m-HAM, Tc99mcolloid.

- Organic radiopharmaceuticals: Tc99m attached to IBI/HMPAO/DTPA/HIDA/ MAG3, I-123-hippuran (see list below, for examples).

Current and future trend is biomolecular radiopharmaceuticals. They interact with cellular molecules at selective sites or along certain biochemical pathways $[1,6]$.

Interactions of biomolecular radiopharmaceuticals (with examples) :

1. Antigen-antibody* : I131-tositumomab with CD20

2. Receptor-ligand* : Y90-DOTATOC with SSTR

3. Transporter-ligand* $:$ I123-FPCIT with DAT

4. Transporter-substrate* : F18-FDG with GLUT
Table 1. List of non-biomolecular vectors and targets

\begin{tabular}{ll}
\multicolumn{1}{c}{ VECTORS } & \multicolumn{1}{c}{ TARGETS } \\
\hline MAG3 & Renal tubules \\
DTPA & Renal glomerules \\
HIDA & Hepatocytes \\
Colloid & Reticuloendothelial system \\
MDP & Bone matrix \\
MAA & Lung capillaries \\
MIBI & Mitochondria \\
HMPAO & Brain cells \\
HAM & Serum, liquor compartements \\
\hline
\end{tabular}

5. Enzyme-substrate*: F18-FDG with hexokinase

6. Enzyme-ligand*: Ga68-TCTP-1 with matrix metalloproteinase-9

The labels determine the use for diagnosis or therapy. Gamma or positron emitters are used for diagnostic purpose, while particulate, nonpositron emitters are for therapy [4].

\section{INSTRUMENTATION}

There are 2 kinds of devices, namely: imaging and counting devices [4].

- Imaging devices. These produce pictorial outputs (images). Nowadays, all devices are digital, such as planar gamma camera, SPECT, and PET. Hybrid systems (SPECT/CT, PET/CT, PET/MRI) precisely localize and define lesions with anatomic landmarks.

- Counting devices. These measure radiation intensity, display numerals or histograms. For examples: probe system, sample counter. They can be connected to Personal Computer.

\section{OUTLINE OF PROCEDURE}

As the roles of NM in diagnosis and therapy, outline of diagnostic and therapeutic procedures are introduced below [4]. 


\section{Diagnostic modality}

Application of a radiopharmaceutical is followed by observation of its biokinetics and functional study of the target organ/body system or characterization and assessment of the lesion viability and targeted biological process [2]. Mode of observation depends on the device used.

- Imaging devices depict temporal distribution of radioavtivity displayed at certain times or over preset time period. Image display may be 2D (static/dynamic image or whole body), 3D (tomogram), 4D (dynamic/ temporal tomogram).

- Counting devices. A probe system is used externally to study invivo function of organs/ body systems. Sample counters such as well-type/liquid scintillation counter are used to measure radiation intensity of biologic samples such as blood, serum or urine. This sample counting method is called radioassay. Invivtro technique determines kinds, quantities, con-centrations of radioactive materials in the biologic samples. It is also called radiobioassay. For example : red cell volume determination. Invitro technique analyzes non radioactive samples by measuring radiation intensity after sample treatment with a radioactive reagent. For example : radioimmunoassay (RIA).

It is necessary to keep in mind that radionuclides for diagnosis and research are ad-ministered in such doses which have no observable adverse biological effect.

\section{Therapeutic modality.}

This modality utilizes the deleterious effect of radiobiologic burden to the lesions. Any lesion must receive its therapeutic dose without significant adverse reactions [7]. In oncology, the doses must be within MTDs (maximal tolerable dose) of critical and dose limiting organs and the administered activities must not exceed MTA (maximal tolerable activity) of individual patients. Hence, accurate theranostic dosimetry prior to each therapy must be carried out to meet the prerequisite. It is the key of personalized medicine. Therapeutic radiopharmaceuticals may be administered directly into or systemically (intravenous, orally) to the lesions [8]. Examples are P-32 ointment applied directly on the skin for keloid and I-131 orally for hyperthyroidism.

\section{NANOTECHNOLOGY}

Nanoparticles (NPs) are vehicles used to facilitate targeting, especially for penetrating biologic barriers such as blood brain barrier. Each NP can be loaded with so many molecules. Single NP may carry molecules of the same target, so targeting effectiveness is enhanced. Radiolabeled NPs can be designed to be able to penetrate cell and nuclear mem-branes, as well as to avoid immune response. Quantum dot (QD), superparamagnetic iron oxide NP (SPION) can be used for cross-validation of bimodal imaging [4,9]. For examples: Cu64QD-RGD for PET versus FMT, In 111-SPIONmesothelin for SPECT versus MRI[10]. Laser (NIR) ir-radiation to AuNP produces heat that can kill or sensitize tumor to radiation, e.g.: Lu177-AuNP-bombesin used for photothermal radiation therapy of prostate cancer. Also, SPION prodoces heat if alternating magnetic field (AMF) is applied extracorporeally. For example: In111-SPION-chL6 for lung cancer. It is called thermomagnetic radiation therapy.

\section{NEUTRON IRRADIATION}

The application of neutron contributes to the advancement of NM. Neutron can play role as an analytic tool or therapeutic weapon against tumor. 


\section{Neutron activation analysis (NAA).}

NAA is an analytic test physically based upon nuclear changes. Capture of neutron by atomic nucleus of the target elements in the sample or body creates unstable radionuclides which revert to a stable condition by the emission of $\geq 1$ gamma rays of characteristic energy. This energy level identifies the element and the level of activities indicates its abundance[11].

\section{Neutron teletherapy.}

Biologic matters contain abundant hydrogen of water. As fast neutron beams collide elastically with tumor hydrogens, then protons recoil, finally stop and emit highly ionizing radiation to sorroundings which kills tumors. This technique is suitable for skin and superficial tumors. For deeper tumor locations, the neutron energy diminishes rapidly along its path to the target, because the body is a good shielding/moderator for neutron radiation. Relative biologic effectiveness (RBE) of neutron is closely related to its kinetic energy: thermal $\approx 1$, epithermal $\approx 2.5$, fast $\approx 5(10-100$ $\mathrm{keV}$ and $2-20 \mathrm{MeV})$ or $\approx 10(0,1-2 \mathrm{MeV})$.

\section{Neutron Capture Therapy.}

In this method, Boron (B-10) is initially carried to a tumor by its oncotropic vector, then the tumor is irradiated with thermal neutron beams producing $\mathrm{Li}-7$ and $\mathrm{He}-4$ ion of 2,3 $\mathrm{MeV}$. He-4 ion is an $\alpha$ particle producing dense ionization along its path. $\mathrm{RBE} \alpha=3-8$. The oncotropic vector may be BSA, BPA, curcuma or NPs loaded with targeting agents [12].

\section{REFERENCES}

1. Feinendegen LE. 1992. Nuclear medicine: a general overview and current trend. In 5th AOCNMB Jakarta-Bali, 25-30 October 1992
2. Tammat SR. 2006. Kimia Radiopharmaka. Workshop nasional preparasi dan aplikasi radiofarmaka. Pusat radioisotop dan radiofarmaka, BATAN

3. Hong H, Zhang Y, Sun J, et al. 2009. Molecular imaging and therapy of cancer with radiolabeled nanoparticles. NanoToday;4:399-413

4. Pranoto S. 2015. Aplikasi kedokteran nuklir. Salatiga: SupraMedika,Ed 3.

5. Wheat JM, Currie GM, Davidson R, et al. 2011. Radionuclide therapy. Radiographer; 58(9):53-61

6. Liu S. 2004. The role of coordination chemistry in the development of targetspecific radiopharmaceuticals. Chem Soc Rev;33:445-461

7. Ersahin D, Doddamane I, Cheng D. 2011. Targeted radionuclide therapy. Cancer;3: 3838-3855

8. Ting G, Chang C-H, Wang H-E, et al. 2010. Nanotargeted radionuclides for cancer nuclear imaging and internal radiotherapy. J Biomed Biotechnol:17pp

9. Morales-Avila E, Ferro-Flores G, OcampoGarcia BE, et al. 2013. Radiolabeled nanoparticles for molecular imaging. http:// www.intechopen.com[10-desember-2013]

10. Misri R, Meier D, Yung AC, et al. 2012. Development and valuation of a dualmodality (SPECT/MRI) molecular imaging bioprobe. Nanomedicine:nanotechnol, biol and med; 8:1007-1016

11. Soenarjo S. 2008. Analisis pengaktipan neutron. $J$ Rad Far;11:37-53

12. Floberg J. 2005. The physics of boron neutron capture therapy: an emerging and in-novative treatment for glioblastoma and melanoma. ] 